11. Hoodless, P. A. et al. MADR1, a MAD-related protein that functions in BMP2 signaling pathways. Cell 85, 489-500 (1996)

12. Eppert, K. et al. MADR2 maps to $18 \mathrm{q} 21$ and encodes a TGF $\beta$-regulated MAD-related protein that is functionally mutated in colorectal carcinoma. Cell 86, 543-552 (1996).

13. Lechleider, R. J., de Caestecker, M. P., Dehejia, A., Polymeropoulos, M. H. \& Roberts, A. B. Serine phosphorylation, chromosomal localization, and transforming growth factor- $\beta$ signal transduction by human bsp-1. J. Biol. Chem. 271, 17617-17620 (1996).

14. Liu, F. et al. A human Mad protein acting as a BMP-regulated transcriptional activator. Nature $\mathbf{3 8 1}$ 620-623 (1996)

15. Hata, A., Lo, R. S., Wotton, D. \& Massagué, J. Mutations increasing autoinhibition inactivate the tumour suppressors Smad2 and Smad4. Nature 388, 82-87 (1997).

16. Baker, J. C. \& Harland, R. M. A novel mesoderm inducer, Madr2, functions in the activin signa transduction pathway. Genes Dev. 10, 1880-1889 (1996).

17. Thiagalingam, S. et al. Evaluation of candidate tumour suppressor genes on the chromosome 18 in colorectal cancers. Nature Genet. 13, 343-346 (1996).

18. Sekelsky, J. J., Newfeld, S. J., Raftery, L. A., Chartoff, E. H. \& Gelbart, W. M. Genetic characterization and cloning of Mothers against dpp, a gene required for decapentaplegic function in Drosophila melanogaster. Genetics 139, 1347-1358 (1995).

19. Savage, C. et al. Caenorhabditis elegans genes sma-2, sma-3 and sma-4 define a conserved family of transforming growth factor $\beta$ pathway components. Proc. Natl Acad. Sci. USA 93, 790-794 (1996).

20. Stura, E. A. \& Wilson, I. A. in Crystallization of Nucleic Acids and Proteins (eds Ducruix, A. \& Giege, R.) 99-126 (Oxford Univ. Press, 1992).

21. Sheldrick, G. in Patterson Interpretation and the Use of Macromolecular Delta-F Data (Daresbury, UK, 1991)

22. Collaborative Computational Project, B. The CCP4 suite: programs for protein crystallography. Acto Crystallogr. D 50, 760-763 (1994)

23. Zhang, K. Y. J. SQUASH-combining constraints for macromolecular phase refinement and extension. Acta Crystallogr. D 49, 213-222 (1993).

24. Jones, T. A., Zou, J.-Y., Cowan, S. W. \& Kjeldgaard, M. Improved methods for building protein models in electron density maps and the location of errors in these models. Acta Crystallogr. A 47, 110-110 (1991).

25. Brunger, A. T. X-PLOR, a system for crystallography and NMR (Yale Univ. Press, New Haven, CT, 1991).

26. Klaulis, P. J. Molscript: a program to produce both detailed and schematic plots of protein structures J. Appl. Crystallogr. 24, 946-950 (1991).

27. Merrit, E. A. \& Murphy, M. E. Raster3D Version 2.0: a program for photorealistic molecular graphics. Acta Crystallogr. D 50, 869-873 (1994).

28. Uchida, K. et al. Somatic in vivo alterations of the JV18-1 gene at 18q21 in human lung cancers. Cancer Res. 56, 5583-5585 (1996).

29. Nicholls, A., Sharp, K. A. \& Honig, B. Protein folding and association: insights from the interfacial an thermodynamic properties of hydrocarbons. Proteins Struct. Funct. Genet. 11, 281-296 (1991).

30. Macias-Silva, M. et al. MADR2 is a substrate of the TGF $\beta$ receptor and its phosphorylation is required for nuclear accumulation and signalling. Cell 87, 1215-1224 (1996).

Acknowledgements. This work was supported by the NIH, the Pew Charitable Trusts, the Arnold and Mabel Beckman Foundation, the Dewitt Wallace Foundation, the Samuel and May Rudin Foundation, and the Howard Hughes Medical Institute.

Correspondence and requests for materials should be addressed to N.P.P. (e-mail: nikola@xray2. mskcc.org). Coordinates are being deposited with the Brookhaven Protein Data Bank.

\section{DNA-replication checkpoint control at the Drosophila midblastula transition}

\section{Ody C. M. Sibon, Victoria A. Stevenson \& William E. Theurkauf}

Department of Biochemistry and Cell Biology and the Institute for Cell and Developmental Biology, State University of New York at Stony Brook, Stony Brook, New York 11794-5215, USA

Embryogenesis is typically initiated by a series of rapid mitotic divisions that are under maternal genetic control ${ }^{1}$. The switch to zygotic control of embryogenesis at the midblastula transition is accompanied by significant increases in cell-cycle length and gene transcription, and changes in embryo morphology ${ }^{2,3}$. Here we show that mutations in the grapes ( $g r p)$ checkpoint 1 kinase homologue ${ }^{4}$ in Drosophila block the morphological and biochemical changes that accompany the midblastula transition, lead to a continuation of the maternal cell-cycle programme, and disrupt DNA-replication checkpoint control of cell-cycle progression. The timing of the midblastula transition is controlled by the ratio of nuclei to cytoplasm (the nucleocytoplasmic ratio), suggesting that this developmental transition is triggered by titration of a maternal factor by the increasing mass of nuclear material that accumulates during the rapid embryonic mitoses ${ }^{5-9}$. Our observations support a model for cell-cycle control at the midblastula transition in which titration of a maternal component of the DNA-replication machinery slows DNA synthesis and induces a checkpointdependent delay in cell-cycle progression ${ }^{10}$. This delay may allow both completion of $S$ phase and transcription of genes that initiate the switch to zygotic control of embryogenesis.

The rapid maternally controlled divisions that initiate embryogenesis in Drosophila melanogaster proceed without cytokinesis, and produce a syncytial blastoderm embryo ${ }^{11}$. We used a screen for mutations that disrupt mitotic chromosome segregation ${ }^{12}$ to identify a maternal-effect mutation, initially designated $f_{s}(A) 4$, that produced severe mitotic defects during the later syncytial divisions.

To analyse directly the effects of this mutation on spindle morphogenesis and cell-cycle progression, we micro-injected embryos derived from homozygous $f_{s}(A) 4$ females with rhodamine-tubulin conjugates, and visualized microtubule reorganization and cell-cycle progression by time-lapse confocal microscopy ${ }^{13,14}$ (Fig. 1). During the final syncytial divisions before the midblastula transition (MBT) (divisions 11-13), the duration of interphase increases from 6 to $14 \mathrm{~min}$ (Table 1). Interphase 14 is prolonged, lasting over $60 \mathrm{~min}$, during which time invaginating membranes surround the majority of nuclei to form a cellular blastoderm $^{11}$. Blastoderm cellularization is the first important morphogenetic event that requires zygotic transcription, and thus cytologically marks the Drosophila $\mathrm{MBT}^{5}$. Our in vivo analyses of the syncytial mitoses demonstrate that embryos derived from homozygous $f_{\mathfrak{s}}(A) 4$ females do not show a significant increase in interphase length during syncytial divisions 11-13 (Fig. 1, Table 1). Furthermore, mutant embryos proceed through at least two extra syncytial cycles of spindle assembly and disassembly and nuclear envelope breakdown and formation after mitosis 13 , and cellularization is never observed (Fig. 1; data not shown). The $f_{s}(A) 4$ mutation thus blocks nearly all of the changes in cell-cycle dynamics and embryo morphology associated with the Drosophila MBT.

The changes in cell-cycle length at the Drosophila MBT are associated with changes in phosphorylation of the cyclin-dependent kinase Cdc2 (ref. 15). Termination of the rapid syncytial divisions normally occurs between 2 and $3 \mathrm{~h}$ after egg deposition, and is accompanied by accumulation of a low electrophoretic-mobility inhibitory tyrosine-phosphorylated form of Cdc2 (ref. 15) (form 4, Fig. 2a). In $f_{s}(A) 4$ mutant embryos, this tyrosine-phosphorylated form of $\mathrm{Cdc} 2$ does not accumulate (Fig. 2a). Studies in yeast and vertebrates indicate that the $\mathrm{Cdc} 2-$ cyclin complex is activated by the Cdc25 phosphatase, which removes the inhibitory tyrosine phosphates and thus drives the cell into mitosis ${ }^{16}$. In Drosophila, a dramatic decrease in the level of String, the Drosophila Cdc25 homologue, accompanies the increase in cell-cycle time at the $\mathrm{MBT}^{15,17}$. In $f_{s}(A) 4$ mutants, String protein levels do not decrease significantly during the later syncytial mitoses (Fig. 2b). The biochemical changes in $\mathrm{Cdc} 2$ that normally accompany the MBT are therefore blocked by the $f_{s}(A) 4$ mutation.

These observations raise the possibility that the $f_{s}(A) 4$ gene product slows the cell cycle by inducing a downregulation of the String phosphatase, thereby allowing accumulation of inhibitory tyrosine-phosphorylated forms of Cdc2. It is also possible that coordinate activation of the Drosophila wee 1 kinase, which is in part responsible for inhibitory tyrosine phosphorylation of $\mathrm{Cdc} 2$ (ref. 18), may also be involved at the MBT.

The MBT is characterized by a sudden increase in zygotic transcription. In Drosophila, the MBT is accompanied by transcription of segmentation genes that are expressed in spatially restricted domains. We therefore used whole-mount in situ hybridization to assay the onset of zygotic segmentation gene expression in $f_{s}(A) 4$ mutant embryos. Before the MBT, the segmentation genes runt, fushi tarazu (Fig. 3) and giant (not shown) are expressed at relatively low levels over broad regions of the embryo ${ }^{19}$, and the early patterns of runt, fushi tarazu and giant expression are observed in $f_{s}(A) 4$ mutants. After mitosis 13, these genes are normally expressed at 
high levels in characteristic stripes ${ }^{19}$ (Fig. 3). In $f_{s}(A) 4$ mutant embryos, by contrast, the post-MBT expression patterns of these genes are not observed (Fig. 3). These data indicate that qualitative changes in zygotic transcription at the MBT are disrupted by the $f_{s}(A) 4$ mutation.

These data indicate that the $f_{s}(A) 4$ mutation blocks nearly all of the changes in embryo morphology, cell-cycle dynamics and biochemistry, and transcriptional regulation that normally accompany the MBT. We therefore conclude that $f_{s}(A) 4$ mutant embryos do not undergo the MBT, but continue to divide under maternal cell-cycle control.

To gain insight into the biochemical function of the gene identified by $f_{s}(A) 4$, we initiated detailed molecular and genetic analyses of this mutation. The $f_{s}(A) 4$ mutation was generated in a Pelement transposon mutagenesis screen ${ }^{20}$. Reversion analyses were performed to determine whether transposon excision could restore $f_{s}(A) 4$ gene function. Most of the P-element excision chromosomes recovered (19 of 26) were homozygous female fertile and complemented the original $f_{s}(A) 4$ mutation $^{20}$. The other excision chromosomes were sterile in combination with the original $f_{s}(A) 4$ allele and when homozygous, suggesting that the transposon excision events had generated deletion alleles. We recovered sequences flanking the $\mathrm{P}$ element in the $f_{s}(A) 4$ stock by the plasmid rescue technique, and used these sequences to localize the insertion to polytene region $36 \mathrm{AB}$ by in situ hybridization. Standard crosses to deficiency chromosomes were then used to confirm that the mutation was located in the $36 \mathrm{AB}$ region (data not shown). These results provided strong genetic evidence that the $f_{s}(A) 4$ mutation was caused by a Pelement transposon insertion into a gene in the $36 \mathrm{AB}$ region of chromosome 2 .

Polytene region $36 \mathrm{AB}$ contains the previously identified maternal-effect lethal mutation grapes $(g r p)^{12,21}$. We therefore tested the $f_{s}(A) 4$ chromosome for the ability to complement $g r p ; f_{s}(A) 4$ fails to complement a P-element-induced loss-of-function allele of grp $\left.(\operatorname{grp})^{I}\right)^{12,21}$. We then used genomic Southern blotting analyses to map the P-element insertion in $f_{s}(A) 4$ to within 300 base pairs of the $\operatorname{grp}^{1}$ P-element insertion (data not shown). Finally, northern blotting with a grp cDNA probe demonstrated that both of the two largest grp transcripts were undetectable in embryos derived from homozygous $f_{\mathcal{S}}(A) 4$ females $^{4}$ (data not shown). Based on these

a

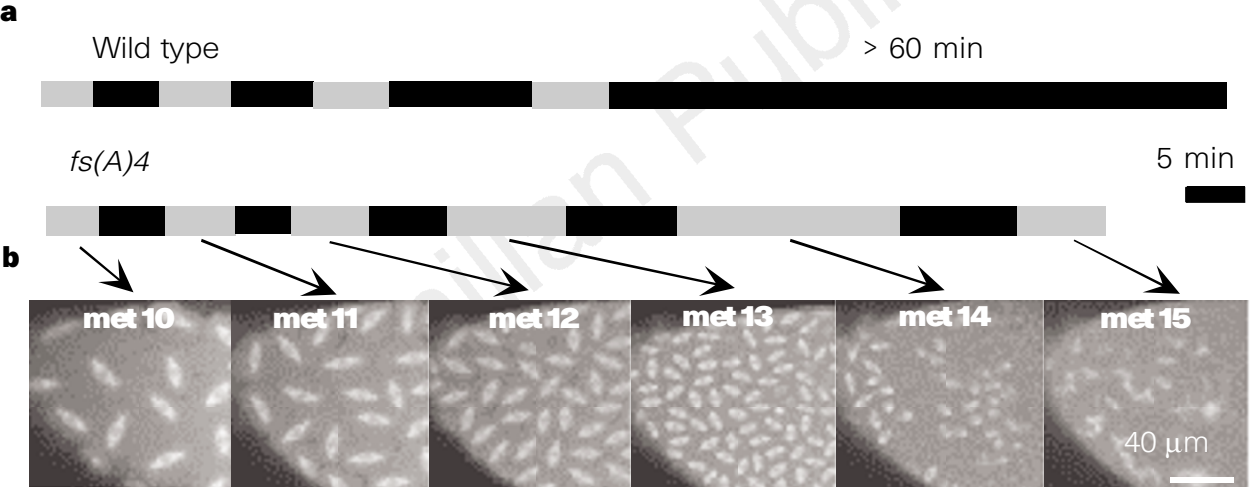

Figure $1 f s(A) 4$ mutant embryos fail to undergo changes in cell-cycle timing and embryo morphology associated with the MBT. a, The bars show the average length of interphase (black) and mitosis (striped) in wild-type and $f_{S}(A) 4$ mutant embryos (scale bar, $5 \mathrm{~min}$; see Table 1). Wild-type embryos show a progressive increase in interphase length during syncytial divisions 11-13, followed by a prolonged interphase 14 of at least $60 \mathrm{~min}$. In $f_{S}(A) 4$ mutant embryos, interphase

a

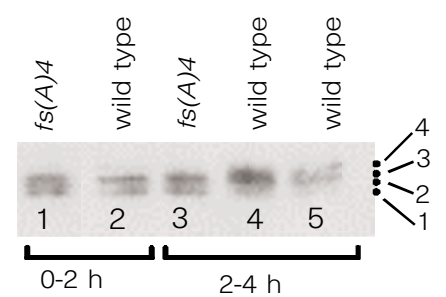

b

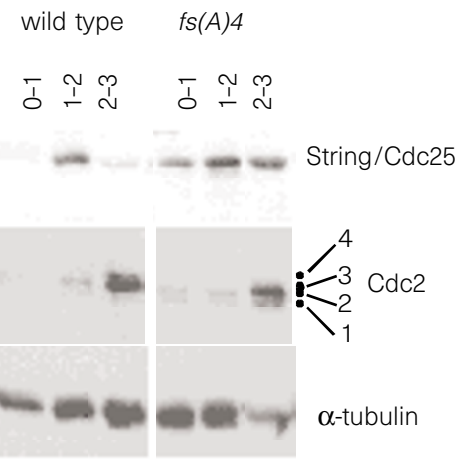

length does not increase significantly during divisions 11-13; mitosis 13 is immediately followed by at least two additional syncytial cycles of spindle assembly/disassembly and nuclear envelope breakdown/assembly. b. Microtubule organization in a living $f_{S}(A) 4$ mutant embryo at metaphase of syncytial cycles 10-15. A time-lapse animation of the continued syncytial divisions in $f_{S}(A) 4$ mutations is available from http://129.49.19.42/biochem/theurkauf.html.

Figure 2 The $f_{S}(A) 4$ mutation prevents inhibitory tyrosine phosphorylation of Cdc2 and String phosphatase downregulation at the MBT. a, Mutant and wildtype embryos $0-2 \mathrm{~h}$ old contain Cdc2 isoforms $1-3$ (see ref. 15). Wild-type embryos 2-4 $\mathrm{h}$ old accumulate a low-mobility tyrosine-phosphorylated form of Cdc2 (form 4, lanes 4,5). Mutant embryos 2-4h old accumulate only trace amounts of this form of Cdc2 (lane 3). b. Wild-type embryos show an increase in String levels during the first $2 \mathrm{~h}$ of development (String/Cdc25, $0-1$ and $1-2 \mathrm{~h}$ ), and a dramatic decrease in String levels as the cell cycle slows at the MBT (2-3 h time point) ${ }^{15}$. The $f_{s}(A) 4$ embryos show the normal increases in String levels between 0 and $2 \mathrm{~h}$, but do not dramatically downregulate String between 2 and $3 \mathrm{~h}$. The middle panels show a duplicate blot probed for Cdc2. The presence of tyrosinephosphorylated Cdc2 form 4 correlates precisely with the decline in String levels in wild-type embryos. The bottom panels show a duplicate western blot probed for $\alpha$-tubulin. 
molecular and genetic studies, we conclude that $f_{s}(A) 4$ is a null or very strong loss-of-function allele of grp, which we designated $\operatorname{grp}^{f(A) 4}$

Loss of grp function has been reported to cause metaphase arrest at division 13 (ref. 21). We observed that $\operatorname{grp}^{f_{s(A) 4}}$ mutant embryos continue through rapid syncytial mitoses and fail to cellularize. To determine whether the continued syncytial divisions and failure to undergo the MBT were specific to the $g r p^{f_{s}(A) 4}$ allele, or were caused by a background mutation on the $g r p^{f_{s}(A) 4}$ chromosome, we analysed embryos derived from $g r p^{f_{s}(A) 4} / g r p^{1}, g r p^{f_{s}(A) 4} / \mathrm{Df}(2 \mathrm{~L}) \mathrm{H} 10$, and $g r p^{1 /}$ $\operatorname{grp}^{1}$ females. The progeny from females of each of these three genotypes showed defects in cell-cycle timing and cellularization that were indistinguishable from those observed in embryos derived from homozygous $\operatorname{grp}^{f_{s}(A) 4}$ females (data not shown). To determine whether these defects were induced by injection of the specific fluorescent probes used in our analyses, we examined embryos injected with rhodamine-tubulin, rhodamine-actin, the chromatin marker oli-green, and a mixture of oli-green and rhodamine tubulin (see Methods). We also assayed cell-cycle progression in uninjected embryos by time-lapse differential interference contrast microscopy. In all cases, we observed identical truncated syncytial cell cycles, additional syncytial mitoses, and a failure of blastoderm cellularization (data not shown). We therefore conclude that the phenotypes described here are not specific to the $\operatorname{grp}^{f_{s}(A) 4}$ allele.

In addition to the cell-cycle defects we report, embryos derived from grp mutant females show mitotic spindle and midbody defects during the later syncytial divisions ${ }^{21}$. We observe cytologically identical defects in spindle assembly in wild-type embryos treated with the DNA-synthesis inhibitor aphidicolin or exposed to X-rays, suggesting that these defects are a secondary consequence of the grp mutation. We believe that these mitotic defects reflect activation of a grp-independent pathway that disrupts the centrosome and blocks spindle function when mitosis is initiated in the presence of damaged or incompletely replicated DNA (O.C.M.S. and W.E.T., manuscript in preparation). Activation of this pathway leads to spindle defects that seem to trigger the spindle checkpoint, leading to increases in the duration of mitosis during the later syncytial divisions in $g r p^{f_{s}(A) 4}$ embryos (Fig. 1). These observations and our analyses lead us to conclude that the primary function of the grp gene product is to control cell-cycle progression in the early embryo, and that loss of grp function leads to continued rapid syncytial mitoses.

We believe that several factors may have obscured the extra syncytial mitoses in grp mutants during earlier analyses ${ }^{21}$. Mitosis 13 in grp mutant embryos is prolonged, and the delay in the onset of anaphase could be mistaken for metaphase arrest (Fig. 1, Table 1). In addition, mitosis 14 and 15 are longer than the intervening interphases, leading to a very high mitotic index in older mutant embryos. Finally, chromosome segregation fails during cycles 1315 , so nuclear density does not increase. Analyses of fixed material and limited in vivo imaging could therefore lead to the conclusion that mutant embryos arrest in metaphase of division 13 (ref. 21), whereas the embryos actually proceed through additional syncytial mitoses.

The grp locus encodes a putative serine-threonine kinase that is most closely related to the check-1 kinase gene $(\operatorname{chk} 1)^{4}$ in Schizosaccharomyces pombe. In fission yeast, the Chk1 protein is in a checkpoint pathway that inhibits cell-cycle progression in response to DNA damage or defects in DNA replication (refs 22, 23 and references therein). To determine whether Grp also lies in a replication checkpoint pathway, we analysed cell-cycle progression directly in living embryos after treatment with the DNA-synthesis inhibitor aphidicolin. For these studies, embryos were co-injected with rhodamine-tubulin and levels of aphidicolin that inhibit DNA synthesis by more than 95\% (ref. 24). Embryos were then monitored by time-lapse confocal microscopy, and the time until

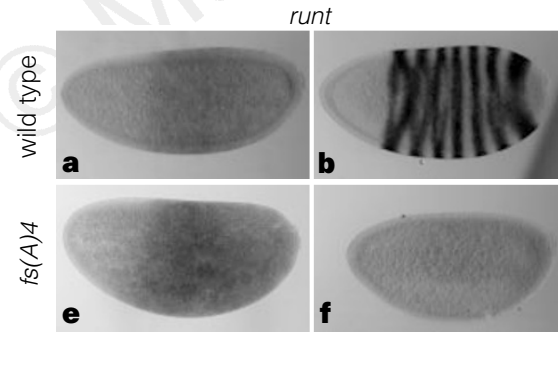

Figure 3 Expression pattern of early zygotic transcripts is impaired in $f_{S}(A) 4$ mutants. Wild-type and $f_{S}(A) 4$ mutant embryos were assayed for expression of early zygotic transcripts from the runt and fushi tarazu genes by whole-mount in situ hybridization. Wild-type embryos before cell cycle 13 show a broad expression pattern of both runt (a) and fushi tarazu (c). After metaphase 13, the

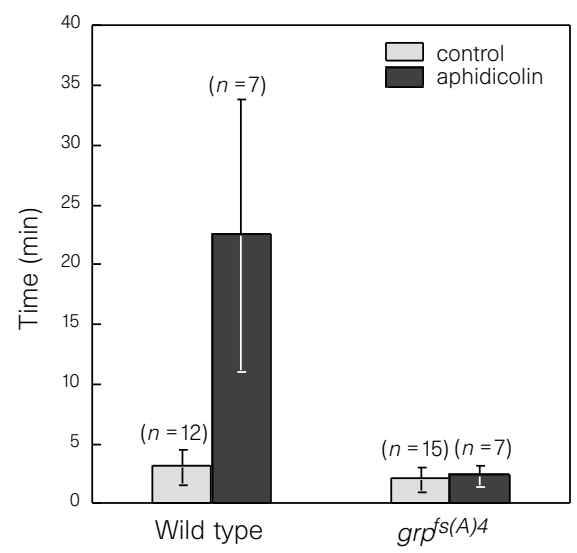

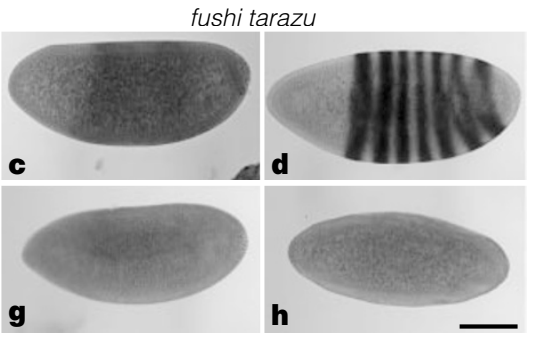

7-striped expression pattern of runt (b) and fushi tarazu (d) are clearly visible ${ }^{19}$. The fs(A)4 mutant embryos show the early broad expression patterns of both runt (e) and fushi tarazu $(\mathbf{g})$, although the latter is less pronounced than in wild-type embryos (compare $\mathbf{g}$ and $\mathbf{c}$ ). By contrast, the 7-striped patterns of expression characteristic of the MBT were never observed in $f S(A) 4$ embryos $(\mathbf{f}, \mathbf{h})$. 


\begin{tabular}{|c|c|c|c|c|c|c|}
\hline \multirow{2}{*}{$\begin{array}{l}\text { Cell cycle } \\
\text { stage } \\
\text { M } 10 \ldots \ldots \ldots \ldots . . . . . . . . .\end{array}$} & \multicolumn{2}{|c|}{$\begin{array}{l}\text { Wild-type } \\
\text { average time } \\
\text { (min) }\end{array}$} & \multirow{2}{*}{$\begin{array}{c}\text { Number } \\
8 \\
8\end{array}$} & \multicolumn{2}{|c|}{$\begin{array}{c}f s(A) 4 \\
\text { average time } \\
\text { (min) }\end{array}$} & \multirow{2}{*}{$\begin{array}{c}\text { Number } \\
6 \\
6\end{array}$} \\
\hline & 5.5 & (0.7) & & 5.6 & (0.4) & \\
\hline | 11 & 6.1 & (1.1) & 9 & 4.7 & (1.0) & 8 \\
\hline M 11 & 5.3 & (0.8) & 10 & 5.4 & $(0.7)$ & 10 \\
\hline 12 & 8.7 & (0.9) & 10 & 6.1 & (1.1) & 11 \\
\hline M 12 & 5.9 & (1.0) & 10 & 6.1 & (1.1) & 12 \\
\hline | 13 & 14.0 & (1.3) & 9 & 7.3 & (1.6) & 12 \\
\hline M 13 & 6.2 & (1.0) & 9 & 10.4 & (2.3) & 10 \\
\hline | 14 & 70.3 & (3.8) & 3 & 11.8 & (3.9) & 8 \\
\hline M 14 & - & - & - & 19.4 & (5.9) & 3 \\
\hline
\end{tabular}

Mean (s.d.) duration of interphase (I) and mitosis (M) stages.

nuclear-envelope breakdown was measured (see Methods). In wildtype embryos during divisions 11 and 12, aphidicolin causes a mean fivefold delay in nuclear-envelope breakdown (Fig. 4). By contrast, grp mutant embryos do not delay nuclear-envelope breakdown in response to aphidicolin treatment, but proceed immediately into mitosis (Fig. 4). These observations indicate that a replication checkpoint pathway functions in the early embryo, and that the grp gene is essential to this pathway. The grp gene is expressed throughout the life cycle of the fly ${ }^{4}$. We have found that the postembryonic development of flies homozygous for the grp mutation is sensitive to hydroxyurea ${ }^{25}$, a feature characteristic of mutations affecting replication checkpoint function (data not shown). The Grp replication checkpoint pathway may therefore serve a nonessential function through most of the life cycle of the fly, but is essential at the MBT.

Our analyses support a simple DNA-replication checkpoint model for cell-cycle control at the $\mathrm{MBT}^{10}$. In this model, a freerunning cell-cycle oscillator drives the rapid divisions that initiate embryogenesis. During the first 10 divisions, maternally supplied components of the DNA synthesis/initiation machinery are in excess, and $\mathrm{S}$ phase is completed before the oscillator triggers mitosis. During divisions 10-13, in contrast, a component of the maternal replication machinery is titrated by the increasing mass of nuclear DNA and becomes rate limiting. DNA synthesis therefore slows, and $S$ phase cannot be completed before the free-running cell-cycle oscillator would normally trigger mitosis. A Grp kinasedependent checkpoint pathway is therefore activated and delays $M$ phase until DNA synthesis is complete. This model is consistent with regulation of the $\mathrm{MBT}$ by the nucleocytoplasmic ratio, explains the absence of a G2 phase and the increase in S phase during the later syncytial mitoses, and accounts for the cell-cycle timing defects observed in grp mutant embryos. Cell-cycle progression in extracts of Xenopus embryos is also dependent on DNA replication ${ }^{10}$, raising the possibility that replication checkpoint control of the MBT is evolutionarily conserved.

The Grp protein could act directly in pathways that induce highlevel zygotic transcription, blastoderm cellularization, and termination of syncytial division at the MBT. However, we favour a model in which the Grp protein functions specifically to control cell-cycle progression in anticipation of the MBT, and the failure to undergo later aspects of MBT in mutant embryos is a consequence of earlier defects in cell-cycle regulation. Consistent with this hypothesis, production of full-length primary transcripts from genes with large transcription units requires the wild-type increases in interphase length that precede the $\mathrm{MBT}^{26,27}$. Further, embryos treated with the transcriptional inhibitor $\alpha$-amanitin do not cellularize after mitosis 13, but proceed through an additional, 14th syncytial mitosis ${ }^{17}$. Finally, the high-level spatially patterned expression of several early zygotic genes is severely impaired or blocked in grp mutant embryos (Fig. 3). These observations suggest a model in which checkpointdependent inhibition of cell-cycle progression during divisions 1013 allows complete transcription of zygotic genes, which in turn act directly to terminate the maternal cell-cycle programme and trigger the transition to zygotic control of embryogenesis.

\section{Methods}

In vivo cytology. For in vivo analyses of the early embryonic divisions, females of the specified genotypes were mated to wild-type Oregon $\mathrm{R}$ males, and the resulting embryos were injected with rhodamine-tubulin (Molecular Probes), rhodamine-actin, or a combination of rhodamine-tubulin and the chromatin marker oli-green (Molecular Probes). Mitosis and cell-cycle progression in injected embryos were assayed by time-lapse confocal microscopy as described for Drosophila oocytes ${ }^{28}$. To quantify cell-cycle phases, interphase was defined by the presence of a nuclear envelope that excluded injected fluorescent protein conjugates, and mitosis was defined by the absence of a nuclear envelope and the presence of spindle structures. To determine the effects of inhibiting DNA synthesis on cell-cycle progression, embryos were injected with a mixture of rhodamine-tubulin $\left(5 \mathrm{mg} \mathrm{ml}^{-1}\right)$ and aphidicolin $\left(100 \mu \mathrm{g} \mathrm{ml}^{-1}\right)$. Microinjection of aphidicolin at this concentration leads to more than $95 \%$ inhibition of DNA synthesis ${ }^{24}$. Breakdown of the nuclear envelope was indicated by entry of rhodamine-tubulin and spindle assembly. Microinjection could not be controlled precisely with respect to cell-cycle phase, so these measurements were standardized as follows. On completion of mitosis, centrosomes duplicate and move to opposite poles of the nuclei, where they remain until nuclear-envelope breakdown and mitosis. We have found that the time between completion of centrosome migration and nuclear-envelope breakdown is consistent from embryo to embryo for a given cell cycle (Fig. 3). Completion of centrosome migration is therefore a consistent cytological marker for mid-interphase. We therefore injected embryos before the centrosomes had completed their movement to the poles, and determined the time between completion of migration and nuclear-envelope breakdown.

In situ hybridization. In situ hybridization for runt, fushi tarazu and giant transcripts was performed as described ${ }^{29}$. Identical probes, hybridization conditions and colour development times were used for wild-type and mutant embryos.

Western blots. For western blot analyses, total protein from staged embryos was subjected to SDS polyacrylamide gel electrophoresis, transferred to membranes, and probed for Cdc2 or String as described ${ }^{15}$. As a loading control, duplicate blots were probed with a monoclonal anti- $\alpha$-tubulin antibody.

Received 24 March; accepted 7 May 1997

1. Gilbert, S. F. Developmental Biology 3rd edn, 75-114 (Sinaur, Sunderland, MA, 1991)

2. Newport, J. \& Kirschner, M. A major developmental transition in early Xenopus embryos: I. characterization and timing of cellular changes at the midblastula stage. Cell 30, 675-686 (1982).

3. Foe, V. E., Odell, G. M. \& Edgar, B. A. in The Development of Drosophila melanogaster (ed Bate, M. \& Martinez Arias, A.) 149-300 (Cold Spring Harbor Laboratory Press, New York, 1993).

4. Fogarty, P. et al. grp, a Drosphila gene with homology ot the S. pombe chk1/rad27 checkpoint gene, is required for the fidelity of the late syncytial divisions. Curr. Biol. (in the press).

5. Edgar, B. A., Kiehle, C. P. \& Schubinger, G. Cell cycle control by the nucleo-cytoplasmic ratio in early Drosophila development. Cell 44, 365-372 (1986).

6. Yasuda, G. K., Baker, J. \& Schubinger, G. Temporal regulation of gene expression in the blastoderm Drosophila embryo. Gen. Dev. 5, 1800-1812 (1991).

7. Pritchard, D. K. \& Schubinger, G. Activation of transcription in Drosophila embryos is a gradual process mediated by the nucleocytoplasmic ratio. Gen. Dev. 10, 1131-1142 (1996).

8. Clute, P. \& Masui, Y. Regulation of the appearance of division asynchrony and microtubuledependent chromosome cycles in Xenopus laevis embryos. Dev. Biol. 171, 273-285 (1995).

9. Kane, D. A. \& Kimmel, C. B. The zebrafish midblastula transition. Development 119, 447-456 (1993).

10. Dasso, M. \& Newport, J. W. Completion of DNA replication is monitored by a feedback system that controls the initiaiton of mitosis in vitro: studies in Xenopus. Cell 61, 811-823 (1990).

11. Foe, V. E. \& Alberts, B. M. Studies of nuclear and cytoplasmic behaviour during the five mitotic cycles that precede gastrulation in Drosphila embryogenesis. J. Cell Sci. 61, 31-71 (1983).

12. Sullivan, W., Fogarty, P. \& Theurkauf, W. E. Mutations affecting the cytoskeletal organization of syncytial Drosophila embryos. Development 18, 1245-1254 (1993).

13. Matthies, H. J., McDonald, H. B., Goldstein, L. S. \& Theurkauf, W. E. Anastral meiotic spindle morphogenesis: role of the non-claret disjunctional kinesin-like protein. J. Cll Biol. 341, 455-464 (1996)

14. Kellogg, D. R., Mitchison, T. J. \& Alberts, B. M. Behavior of microtubules and actin filaments in living Drosophila embryos. Development 103, 675-686 (1988).

15. Edgar, B. A., Sprenger, F., Duronio, R. J., Leopold, P. \& O'Farrell, P. H. Distinct molecular mechanisms regulate cell cycle timing at successive stages of Drosophila embryogenesis. Gen. Dev. 8, 440-452 (1994)

16. Elledge, S. J. Cell cycle checkpoint: preventing an identity crisis. Science 274, 1664-1672 (1996).

17. Edgar, B. A. \& Datar, S. A. Zygotic degradation of two maternal Cdc 25 mRNAs terminates Drosophila's early cell cycle program. Gen. Dev. 10, 1966-1977 (1996).

18. Campbell, S. D., Sprenger, F., Edgar, B. A. \& O'Farrel, P. H. Drosophila Wee 1 kinase rescues fission yeast from mitotic catastrophe and phosphorylates Drosophila Ccd2 in nitro. Mol. Biol. Cell 6, 13331347 (1995).

19. Klinger, M. \& Gergen, J. P. Regulation of runt transcription by Drosophila segmentation genes. Mech. Dev. 43, 3-19 (1993).

20. Cooley, L., Kelley, R. \& Spradling, A. Insertional mutagenesis of the Drosophila genome with single P elements. Science 239, 1121-1128 (1988) 
21. Fogarty, P., Kalpin, R. F. \& Sullivan, W. The Drosophila maternal-effect mutation grapes causes metaphase arrest at nuclear cycle 13. Development 120, 2131-2142 (1994)

22. Francesconi, S., Grenon, M., Bouvier, D. \& Baldacci, G. p56 $6^{\text {chk1 }}$ protein kinase is required for the DNA replication checkpoint at $37^{\circ} \mathrm{C}$ in fission yeast. EMBO J. 16, 1332-1341 (1997).

23. Walworth, N. C. \& Bernards, R. rad-dependent response of the chk1-encoded protein kinase at the DNA damage checkpoint. Science 271, 353-356 (1996).

24. Raff, J. W. \& Glover, D. M. Nuclear and cytoplasmic cycles continue in Drosophila embryos in which DNA synthesis is inhibited with aphidicolin. J. Cell Biol. 107, 2009-2019 (1988).

25. Banga, S. S., Shenkar, R. \& Boyd, J. B. Hypersensitivity of Drosophila mei-41 mutants to hydroxyurea is associated with reduced mitotic chromosome stabiity. Mutant. Res. 163, 157-165 (1986).

26. Shermoen, A. W. \& O'Farrell, P. H. Progression of the cell cycle through mitosis leads to abortion of nascent transcripts. Cell 67, 303-310 (1991)

27. Ruden, D. M. \& Jackle, H. Mitotic delay dependent survival identifies components of cell cycle contro in the Drosophila blastoderm. Development 121, 63-73 (1995).

28. Theurkauf, W. E. in Methods of Cell Biology: Drosophila melanogaster: Practical Uses in Cell and Molecular Biology (eds Goldstein, L. S. B. \& Fryberg, E.) 489-505 (Academic, New York, 1994).

29. Tautz, D., Pfeifle, C. A non-radioactive in situ hybridization method for the localization of specific RNA's in Drosophila embryo's reveals translational control of the segmentation gene hunchback. Chromosoma 98, 81-85 (1989).

Acknowledgements. We thank W. Sullivan, S. Campbell, B. Holdener, J. Kramer, N. Hollingsworth and B Eggen for comments on the manuscript; B. Edgar and P. O'Farrell for discussions; P. Gergen for probe and advice on whole-mount in situ hybridization; P. Fogarty and W. Sullivan for sharing unpublished dat and providing grp cDNA clones; R. S. Hawley and colleagues at the University of California at Davis for providing their collection of P-element-associnted maternal-effect lethal mutations; and B. Edgar for anti-

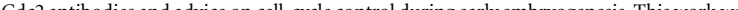
a fellowship from the NWO to O.C.M.S. and grants from the NIH and American Cancer Society to W.E.T

\section{Structure of the multimodular endonuclease Fokl bound to DNA}

\section{David A. Wah, Joel A. Hirsch*, Lydia F. Dorner ${ }^{\star}$ Ira Schildkraut $\dagger$ \& Aneel K. Aggarwal ${ }^{\star}$}

Department of Biochemistry and Molecular Biophysics, Columbia University, New York, New York 10032, USA

$\dagger$ New England Biolabs, 32 Tozer Road, Beverly, Massachusetts 01915, USA

FokI is a member of an unusual class of bipartite restriction enzymes that recognize a specific DNA sequence and cleave DNA nonspecifically a short distance away from that sequence ${ }^{1-3}$. Because of its unusual bipartite nature, FokI has been used to create artificial enzymes with new specificities ${ }^{4-7}$. We have determined the crystal structure at $2.8 \AA$ resolution of the complete FokI enzyme bound to DNA. As anticipated, the enzyme contains amino- and carboxy-terminal domains corresponding to the DNA-recognition and cleavage functions, respectively. The recognition domain is made of three smaller subdomains (D1, D2 and D3) which are evolutionarily related to the helix-turn-helixcontaining DNA-binding domain of the catabolite gene activator protein CAP $^{8}$. The CAP core has been extensively embellished in the first two subdomains, whereas in the third subdomain it has been co-opted for protein-protein interactions. Surprisingly, the cleavage domain contains only a single catalytic centre, raising the question of how monomeric FokI manages to cleave both DNA strands. Unexpectedly, the cleavage domain is sequestered in a 'piggyback' fashion by the recognition domain. The structure suggests a new mechanism for nuclease activation and provides a framework for the design of chimaeric enzymes with altered specificities.

FokI exists as a monomer and recognizes an asymmetric DNA sequence 5'-GGATG-3', cleaving DNA phosphodiester groups 9 and 13 base pairs (bp) away from the recognition site (Fig. 1). The structure shows the enzyme approaching DNA from the majorgroove side, where it appears to surround the DNA (Fig. 2). Subdomain D1 of the recognition domain covers the DNA major

* Present addresses: Department of Molecular Biophysics and Biochemistry, Yale University, New Haven, Connecticut 06511, USA (J.A.H.); Department of Physiology and Biophysics, Mount Sinai School of Medicine, New York, New York 10029, USA (A.K.A.). groove, recognizing base pairs at the $3^{\prime}$ end of the recognition sequence (GGATG). D1 is a compact structure of eight helices $(\alpha 1$ to $\alpha 8$ ), two loops (L1 and L2), a $\beta$-sheet ( $\beta 1$ to $\beta 3$ ) and an Nterminal arm (Fig. 3a). Helices $\alpha 4, \alpha 5$ and $\alpha 6$ and loops L1 and L2 make up the modified helix-turn-helix (HTH) motif. The short helices $\alpha 4$ and $\alpha 5$ share the same helical axis, as if they were part of a single $\alpha$-helix into which L1 has been inserted. Together, $\alpha 4$ and $\alpha 5$ $(\alpha 4 / 5)$ form the first helix of the HTH motif; $\alpha 6$ is the second helix (also known as the recognition helix) which lies against the DNA major groove. The turn expected between $\alpha 4 / 5$ and $\alpha 6$ is replaced by L2. Both loops reach out like fingers from the helices to make basespecific contacts with the DNA. D1 connects to D2, which contacts base pairs at the $5^{\prime}$ end of the recognition sequence (GGATG). D2 is an extended, triangular-shaped structure of six helices ( $\alpha 1$ to $\alpha 6$ ), a $\beta$-sheet $(\beta 1, \beta 2, \beta 5)$, a $\beta$-hairpin $(\beta 3, \beta 4)$, and a short loop L1 (Fig. $3 a)$. As in D1, the basic HTH motif is extensively modified. $\alpha 2$ and $\alpha 5$ comprise the two $\alpha$-helices of the HTH motif, with $\alpha 5$ lying in the DNA major groove. The 'turn' is replaced by loop L1, a pair of antiparallel helices $\alpha 3$ and $\alpha 4$, and a short segment T1 connecting $\alpha 4$ to $\alpha 5$. D3 is most similar to CAP and related proteins such as histone $\mathrm{H} 5, \mathrm{HNF}-3 \gamma$ and the biotin operon repressor $(\mathrm{BirA})^{8-11}$ (Fig. 3a). Despite this similarity, D3 barely touches the DNA. Remarkably, its recognition helix lies outside the DNA major groove and is used primarily to piggyback the cleavage domain onto the recognition domain. Thus, we observe an almost identical $\mathrm{HTH}$ motif being used in a different context. In the CAP-related protein-DNA complexes, the motif is used to recognize the DNA bases, whereas in D3 the motif is used primarily to mediate proteinprotein interactions.

As already noted, subdomains D1 and D2 make almost all of the base-specific contacts with the DNA. The DNA maintains a B-form DNA conformation without any major bends or kinks. Interactions between D1 and DNA occur along four segments of the subdomain. The N-terminal arm, loop L1, and the recognition helix $\alpha 6$ form specific contacts in the major groove, whereas loop L2 makes contacts in the minor groove. D2, on the other hand, contacts DNA exclusively in the major groove by $\alpha 4$ and T1 of the 'turn', and the recognition helix $\alpha 5$. The recognition helices of D1 and D2 approach DNA differently. The D1 recognition helix is most similar to the canonical form, packing against the major groove with its $\alpha$ helical axis roughly perpendicular to the DNA axis ${ }^{12}$. In contrast, the recognition helix of D2 juts away from the DNA, its $\alpha$-helical axis tilted by $\sim 35^{\circ}$ with respect to the plane of the base pairs. The 'angle of attack' is reminiscent of the way the $\alpha$-helices of the zinc-fingers of protein Zif268 approach DNA ${ }^{13}$. As a unit, the HTH of D2 is flipped $180^{\circ}$ with respect to the HTH of D1, analogous to the inverse orientations of the HTH motifs of the homeodomain and the POUspecific domain of the Oct-1 POU domain ${ }^{14}$.

The protein-DNA interactions are extensive. For instance, almost all of the hydrogen-bond donor and acceptor groups in the major groove of the recognition sequence are involved in direct contacts with the protein. This complementarity at the proteinDNA interface ensures that only the FokI recognition sequence can form all of the interactions. The three guanines of the recognition sequence (GGATG) make bidentate hydrogen bonds with arginine and lysine residues, and the adenines of the third and fourth base

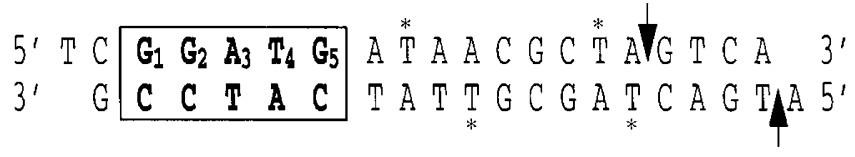

Figure 1 Sequence of the 20-bp DNA fragment used to co-crystallize FokI. The recognition sequence is numbered and the sites of cleavage are indicated by arrows. Asterisks denote the thymine residues substituted by iodouracils in the derivative lodo (Table 1). This oligomer can be cleaved by the enzyme (data not shown). 